\title{
Aspectos sanitários da pecuária leiteira na região do Médio Paraíba - Rio de Janeiro e Minas Gerais
}

\section{Sanitary aspects of dairy cattle in the region of medium Paraíba - Rio de Janeiro and Minas Gerais}

\author{
Rita de Cássia Campbell Machado Botteon, “ Paulo de Tarso Landgraf Botteon, ${ }^{\star \star}$ Zelson Giácomo Lóss ${ }^{\star \star \star ~}$
}

\begin{abstract}
Resumo
A pecuária leiteira constitui uma das mais importantes atividades econômicas do país. Estudos para identificação de causas e índices de mortalidade são raros. As principais causas de enfermidades relacionam-se ao manejo e condições precárias de higiene alimentar e ambiental. A presente investigação foi empreendida objetivando o reconhecimento de aspectos relacionados à sanidade do rebanho bovino leiteiro na região do Médio Paraíba - RJ e MG. De uma relação de Unidades de Produção de Leite C (UPL), um questionário foi aplicado a 32 propriedades em agosto de 1998 e reaplicado em julho de 1999 às 20 UPL cujas informaçōes permitiram o preenchimento completo do formulário proposto. Todos os entrevistados informaram contar com Assistência Veterinária. Quanto ao manejo profilático, as práticaș mais difundidas foram vacinação, vermifugação e controle de carrapatos. A maioria informou a preferência por produtos injetáveis. A forma mais freqüente de higienização dos tetos e quartos mamários antes e após a ordenha, consistiu em deixar o bezerro mamar. A identificação de mastite foi preocupação pouco comum. Corte e desinfecção do cordão umbilical de forma sistemática não foram informados por nenhum dos entrevistados. Em todas as UPL o destino dos resíduos orgânicos foi uma capineira, horta ou jardim. Três criadores informaram enterrar os cadáveres ocasionalmente. As medidas higiênico-sanitárias informadas foram pouco efetivas, ainda que freqüentes.
\end{abstract}

Palavras-chave: pecuária leiteira, sanidade, bovinos.

\begin{abstract}
The dairy cattle constitutes one of the most important economic activities of the country. Studies for identification of causes and mortality rate are rare. The main causes of illness are related to the handling and precarious hygienic conditions of food and environmental. The present inquiry was undertaken with the purpose to recognize aspects related to the sanity of dairy cows in the area of Medium Paraíba - RJ and MG. Of a report of Units of Production of milk C (UPM), a questionnaire was applied to 32 properties in August of 1998 and reapplied in July of 1999 to 20 UPM whose information allowed the complete filling of the proposed formulary. The interviewers informed to count with Veterinary Attendance. In relation to the prophylactic handling the spread practices were vaccination and control of worms and ticks. Most informed the preference for injectable products. The most frequent form of hygienization of the mammary glands before and after it milks consisted in leaving the calf to suckle. The mastitis identification was not a very common concern. The desinfection of the umbilical cord in a systematic way was not informed by interviewers. In all the UPM the destiny of the organic residues was a pasture, vegetable garden or garden. Three owners informed to bury the cadavers occasionally. Hygienic sanitary measures were not very effective although they were
frequently.
\end{abstract}

Keywords: Dairy cattle, sanity, bovine.

\section{Introdução}

A análise da história recente da pecuária leiteira no Brasil mostra uma contradição. A década de 1990 apresentou avanços em produção, tecnologia e qualidade, mas conviveu com a crescente produção de leite informal e importações que emperraram o avanço pretendido. Segundo a Associação Brasileira dos Produtores de Leite, enquanto o preço do !eite pago ao produtor caiu, o setor obteve aumento de produtividade. Segundo dados do IBGE, em muitos aspectos de produção a pecuária leiteira no Brasil compara-se à Idade Média. Um grande número de pequenos produtores operam com baixa produtividade e têm participação decrescente na oferta global de leite. A maioria desses produtores encontra-se com dificuldades, produzindo leite caro, recebendo pouco pelo leite produzido e continua resistindo, apesar da precariedade \footnotetext{
* Departamento de Medicina e Cirurgia Veterinária (DMCV) - Instituto de Veterinária (IV) Universidade Federal Rural do Rio de Janeiro (UFRRJ)
Caixa Postal 74562 cep 23851-970 - Seropédica-RJ e-mail rbotteon@uírri.br

** DMCV-IV-UFRRJ. pbotteon@ufrrj

*** DMCV -IV - UFRRJ.
} 
das condições e dos prejuizos sofridos (Prado, 1991; Viana et al., 1987; Lima, Leite, 1982). No Brasil, estudos para identificação de causas e indices de mortalidade são raros e a literatura é particularmente escassa. Alguns estudos têm demonstrado elevada prevalência de enfermidades em diferentes condições de manejo (Moreira, 1988; Langeneger et al., 1974; Figueiredo, 1988, Prado, 1991; Vianna et al., 1987; Lima, Leite, 1982). As principais causas de enfermidades estão relacionadas ao manejo inadequado e precárias condições de higiene alimentar e ambiental como veiculadores de doenças. A criação bem-sucedida baseia-se num bom manejo, assim como nas boas normas de nutrição e higiene (Lucci, 1989). Diante da complexidade de fatores envolvidos, prevenção é a palavra-chave. As bases da prevenção dizem respeito à ingestão adequada de colostro, reforço da imunidade específica e redução do aporte de agentes infecciosos (Lucci, 1989; Hall et al., 1992; Smith, 1993).

A presente investigação foi empreendida com o objetivo de contribuir para o esclarecimento de fatores envolvidos na ocorrência de enfermidades e o reconhecimento de aspectos relacionados às condições de criação e produção dos rebanhos.

\section{Material e métodos}

De uma relação de Unidades de Produção de Leite tipo C (UPL), localizadas entre os municípios de Valença, Rio das Flores e Rio Preto (RJ e MG), foram selecionadas 32 propriedades, nas quais um questionário para avaliação das condições higiênico-sanitárias foi aplicado através de entrevista pessoal em agosto de 1998. O mesmo questionário foi reaplicado em julho de 1999 às 20 UPL cujas informações permitiram o preenchimento completo do formulário proposto inicialmente. Contribuíram para a seleção das UPL a serem estudadas, as condições de acesso, a proximidade entre os grupos e, sobretudo, a concordância do proprietário em fornecer com fidelidade as informações pretendidas.

\section{Resultados e discussão}

Questionários para avaliação das condições sanitárias aplicados às 20 UPL, foram respondidos por 11 proprietários, seis administradores e três encarregados. Todos os entrevistados informaram contar com Assistência Veterinária, em freqüência semanal $(10 \%)$, quinzenal $(15 \%)$, mensal $(30 \%)$ ou ocasional (45\%). A maioria (85\%) informou que a assistência veterinária thes era proporcionada por convênio com o Laticínio ou Cooperativa aos quais forneciam o leite. Três criadores informaram assistência particular. Como assistência ocasional, $45 \%$ dos entrevistados referiram-se a situações de risco para a vida do animal ou situações não resolvidas com práticas de rotina.

Quanto ao manejo profilático de doenças, a prática mais difundida entre os criadores foi a vacinação. Vacinação contra febre aftosa, foi informada em $100 \%$ das UPL estudadas em semelhança com os dados obtidos por Moreira (1988) em estudo semelhante no município de João Pinheiro (MG). Vacina contra raiva foi aplicada por $95 \%$ dos proprietários entrevistados. Utilização de vacinas contra brucelose e carbúnculo sintomático foram relatadas por $50 \%$ e $30 \%$ dos criadores. O índice de vacinação contra brucelose de $25 \%$ descrito por Leite, Lima (1982) em Minas Gerais foi inferior, enquanto o índice de $77 \%$ relatado por Langenegger et al. (1974) no Rio de Janeiro foi superior ao obtido neste estudo. Dois proprietários informaram a utilização de vacinas contra vibriose e 3 contra leptospirose. Pneumoenterite passou a ser prevenida com a aplicação de vacina em uma propriedade (5\%). Em relação às vacinações contra raiva, vibriose e leptospirose, os resultados obtidos se assemelham aos relatos de Prado (1991).

Treze entrevistados (65\%) informaram a utilização de produtos carrapaticidas mediante a presença de animais altamente parasitados. Os demais (35\%) informaram a utilização em intervalos menores que permitiram até 6 aplicações anuais. Dos proprietários que informaram medidas ocasionais para controle de carrapatos, $3(15 \%)$ informaram a utilização de produtos tópicos na forma "Pour on", 5 (25\%) a utilização de banhos de aspersão, e os demais (25\%) informaram utilizar produtos injetáveis. Os entrevistados que informaram aplicações mais freqüentes informaram a preferência por produtos injetáveis. As bases farmacológicas utilizadas por $85 \%$ dos entrevistados foram Ivermectina e Amitraz. A ampla utilização de produtos carrapaticidas com preferência por produtos de aplicação tópica foi relatada por Leite, Lima (1982) e Prado (1991) em Minas Gerais. Intervalo de aplicação inferior a 30 dias relatados por Prado (1991) e Viana et al. (1987) foram menos freqüentes no presente trabalho. Um proprietário informou a utilização de subdosagem de Ivermectina em intervalos aproximados de 30 dias.

O combate aos bernes em todas as UPL foi descrito como ocasional e o produto mais utilizado foi a mistura de óleo queimado com Triclorfon em aplicação tópica. Dados semeIhantes foram relatos por Leite, Lima (1982) e Prado (1991). A maioria dos entrevistados informou que a aplicação de Ivermectina resulta em controle eficaz do berne. A maioria dos entrevistados (55\%) informou a utilização de Ivermectina em animais adultos em intervalos variáveis e o fornecimento do leite à cooperativa ou laticínio sem respeitar o período de carência para o consumo.

Nenhuma forma de higienização dos tetos e quartos mamários antes e após a ordenha foi informada por $35 \%$ dos entrevistados. Higiene com água $(50 \%)$ ou água clorada $(15 \%)$ foi informada em $13(65 \%)$ UPL. As práticas descritas diferiram daquelas relatadas por Prado (1991) e Viana et al. (1987), em Sete Lagoas - MG.

Utilização de iodo após a ordenha como forma de prevenção de mastite foi restrita a quatro propriedades que utilizavam ordenha mecânica e duas que realizavam ordenha mecânica e manual seletivamente (mista). Em três UPL que realizavam ordenha mista, não foram descritas formas específicas de higiene após a ordenha. A forma mais freqüente de higienização após a ordenha (55\%) consistiu em deixar o bezerro fazer o "repasse".

A identificação de mastites não se caracterizou como preocupação entre os criadores. A maioria ( $85 \%$ ) informou identificar mastite pelas alterações na glândula mamária ou composição do leite. "Quebra no leite" foi informada por dois encarregados como sugestiva de mastite. Três criadores (15\%) informaram realizar testes para identificação de mastite subclínica periodicamente. Prado (1991) relatou que 30\% dos criadores asseguravam não serem capazes de identificar mastite. 
Os procedimentos após identificação de casos clínicos foram pouco variados e a maioria $(60 \%)$ informou tratar imediatamente com uma ou duas aplicações intramamárias do produto disponível no momento. A maioria informou "tentar" mais de um produto antes de solicitar a presença do Médico Veterinário. Em 30\% das UPL, além de tratar, os animais com mastite clínica passaram a ser ordenhados por último.

A maioria dos criadores (70\%) dispensaram cuidados mais intensivos à higiene dos currais e salas de ordenha que às instalações destinadas aos bezerros. Nestas propriedades, em geral, a higiene dos currais e salas de ordenha fora realizada diariamente, na forma de varredura e lavagem, enquanto que para a higiene dos bezerreiros prevaleceu em $65 \%$ das criações, a raspagem com enxada ou varredura. Em três propriedades foram identificados cuidados intensivos com as instalações destinadas aos bezerros. Caiação foi relatada de forma ocasional em duas propriedades.

Corte e desinfecção do cordão umbilical de forma sistemática não foram informados por nenhum dos entrevistados. Nenhuma forma de desinfecção foi relatada por quatro entrevistados. A maioria $(60 \%)$ informou a utilização de iodo ou spray "mata bicheira" ocasionalmente. A freqüência de corte e desinfecção do umbigo relatada foi inferior à descrita Prado (1991), onde $30 \%$ do total de criadores utilizava iodo como medida preventiva de infecções umbilicais e $88 \%$ realizavam corte do umbigo. No presente trabalho, creolina e ungüento

\section{Referências}

Anuário estatístico do IBGE. 1989.

FIGUEIREDO, M.C.P. Alguns aspectos da situação sanitária bovina do município de Uberaba, Minas Gerais. 1979. 172 p. Tese (Mestrado). Escola de Veterinária - Universidade Federal de Minas Gerais.

HALL,G.A; JONES,P.W.; MORGAN,J.H. Calf diarrhea. In: ANDREW, BLOWEY, BOYD \& EDDY. Bovine Medicine. 1992. p. 154-180.

LANGENEGGER, J.; COELHON.M.; MENKE, L.G. Manejo deficiente, o maior problema da criação de bezerros. Biol. Campo. Rio de Janeiro. v. 262 , n. 11 , p. $60-64,1974$

LEITE, R.C.; LIMA, J.D. Fatores sanitários que influenciam na criação de bezerros. Arq. Esc. Vet. UFMG, Belo Horizonte, v. 34, n. 3, p. 485492, 1982. para cura do umbigo foram citados em uma propriedade cada O proprietário que informou a utilização de creolina informou ainda a aplicação de duas gotas do mesmo produto na narina "para estimular a respiração". Leite Lima (1982) verificaram que $83,3 \%$ dos criadores de Sete Lagoas realizavam a cura do umbigo. Índices semelhantes foram descritos por Langenegger et al. (1974), no Rio de Janeiro.

Em todas as UPL o destino dos resíduos orgânicos foi capineira, horta ou jardim. Esterqueira foi utilizada em três propriedades. Na maioria, os resíduos orgânicos foram amontoados próximo ou jogados diretamente na capineira. Quanto ao destino dos animais mortos, $75 \%$ dos entrevistados informaram que os cadáveres são jogados no rio ou deixados para os urubus. Em três UPL (15\%) os cadáveres foram enterrados ocasionalmente.

\section{Conclusões}

Ainda que vacinações e controle parasitário tenham sido relatados pela maioria dos entrevistados, as formas e os critérios de utilização de produtos, bem como as medidas higiênicas adotadas foram pouco adequadas e realizadas com poucos critérios técnicos, ainda que todos os criadores contassem com assistência veterinária. Observações pessoais permitem avaliar que as medidas adotadas se mantêm mais por força da tradição que por falta de informação.

LUCCI, C. Bovino leiteiros jovens: nutrição, manejo e doenças. São Paulo: Nobel, 1989.

MOREIRA, F. X. Condições sanitárias e estrutura de produçāo na bovinocultura dos núcleos de João Pinheiro e Rio Verde no estado de Minas Gerais. 1988. 92 p. Tese (Mestrado). Escola de Veterinária - Universidade Federal de Minas Gerais.

PRADO, E. Características socioeconômicas e sanitárias da pecuária bovina do município de Divinópolis, Minas Gerais. 1991. 205 p. Tese (Mestrado), Escola de Veterinária - Universidade Federal de Minas Gerais.

SMITH, B.P. Tratado de medicina interna de grandes animais. São Paulo: Manole, 1993.

VIANA, F. C., CRUZ, F. E. R., LAENDER, F. C. et al. Diagnóstico da situação de produção bovina de leite do município de Sete Lagoas MG. Arq. Bras. de Med. Vet. e Zoot., v. 39, n. 5, p. 699-717, 1987. 